\title{
Lena, une référence en traitement d'images
}

François Lecellier et Christine Fernandez-Maloigne

\section{(2) OpenEdition}

\section{Journals}

Édition électronique

URL : http://journals.openedition.org/itti/1146

DOI : 10.4000/itti. 1146

\section{Éditeur}

Université de Poitiers

\section{Référence électronique}

François Lecellier et Christine Fernandez-Maloigne, «Lena, une référence en traitement d'images », Images du travail, travail des images [En ligne], 3 | 2017, mis en ligne le 01 février 2017, consulté le 14 avril 2021. URL : http://journals.openedition.org/itti/1146 ; DOI : https://doi.org/10.4000/itti.1146

Ce document a été généré automatiquement le 14 avril 2021.

Images du travail, travail des images 


\section{Lena, une référence en traitement d'images}

\section{François Lecellier et Christine Fernandez-Maloigne}

Image originale de Lena.

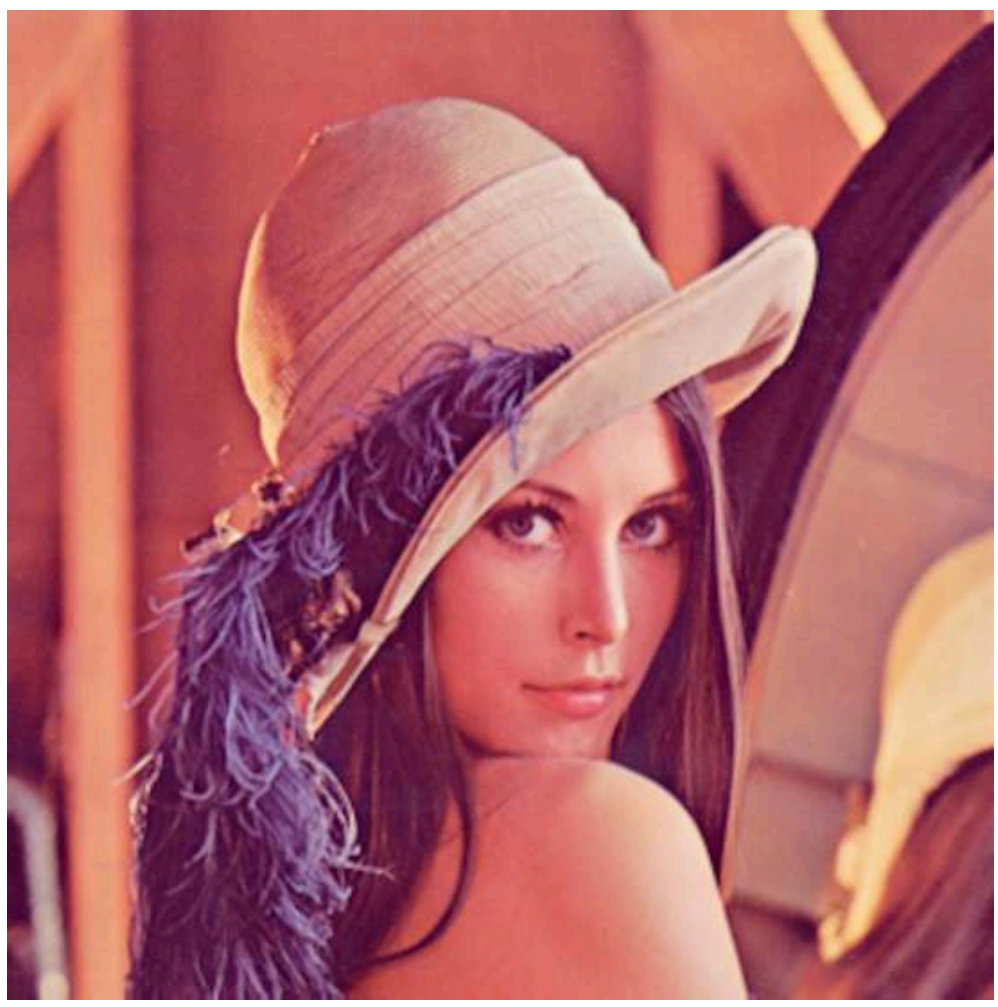

Afin de tester les différents algorithmes développés, les traiteurs d'images ont besoin d'images comportant des caractéristiques bien particulières. L'une des images les plus utilisées depuis 1973 s'intitule Lena et comporte un mélange de détails, d'aplat de couleurs, de textures et d'ombres et permet de tester une grande variété d'algorithmes de filtrage, de segmentation ou de compression. C'est certainement l'une des premières 
images présentée aux étudiants ou au public quand on parle de traitement d'images et par cette célébrité, on la surnomme " La première dame d'Internet ".

2 Mais cette image n'a pas été créée par des chercheurs pour cette utilisation, elle a été scannée en 1972 depuis une image de plus grande dimension. L'image coupée, désormais de référence, représente le visage d'une jeune femme, un chapeau sur la tête, l'épaule visible tournant à moitié le dos à un miroir. Elle est de dimension 512x512 pixels et a donc été obtenue après avoir coupé la version initiale d'un portrait de femme au niveau des épaules. Cette image de départ, toujours disponible sur Internet, provient d'une source inattendue pour le traitement d'images: la page centrale du numéro de novembre 1972 du magazine masculin Playboy!

3 La première personne ayant décidé d'utiliser ce portrait comme image de test faisait partie d'une équipe de chercheurs de l'institut de traitement du signal et des images de l'université de Californie du Sud qui recherchaient une image avec un visage humain, des détails et des textures. Il parait clair que l'utilisation de cette image dans un milieu très largement masculin ne tient pas uniquement à ses caractéristiques objectives, mais que les chercheurs préfèrent travailler sur une image attrayante, y compris dans sa version tronquée.

4 Cette image est importante pour la communauté de par sa grande variabilité et son contenu diversifié. En effet, lors des tests des algorithmes développés en traitement d'images, il est toujours nécessaire de vérifier s'ils peuvent s'adapter à la majorité des types d'images. Lena présente plusieurs zones texturées au niveau des cheveux, du chapeau et des plumes ornant ce dernier. Le fond de l'image est composé de plusieurs zones distinctes légèrement floutées et le visage et l'épaule proposent des zones d'aplat et d'ombre particulièrement intéressantes pour vérifier les dégradations possibles des algorithmes de compression par exemple. Il est à noter que l'image est très souvent utilisée dans sa version en niveau de gris du fait de sa faible variabilité au niveau des couleurs et que les traiteurs d'images spécialisés dans le domaine de la couleur utiliseront d'autres exemples pour vérifier la fiabilité de leurs algorithmes.

Mais l'image est à présent un standard dans le domaine et continue à être largement utilisée même 43 ans après sa sortie, sans avoir pris une ride !

\section{AUTEURS}

\section{FRANÇOIS LECELLIER}

François Lecellier est Maître de Conférences en informatique et applications et a obtenu sa thèse de doctorat en 2009 à l'Université de Caen sur des problématiques de segmentation d'images médicales. Après un an de post-doctorat à l'INRIA de Rennes, il a été recruté au laboratoire Xlim au sein de l'équipe Icones. Depuis, il travaille sur la thématique de l'indexation automatique ou semi-automatique des images et des vidéos. Responsable communication de la fédération de recherche MIRES et du programme NUMERIC du CPER, il est également responsable scientifique de plusieurs actions de recherches transverses de l'Université de Poitiers, de projets CNRS ou 
d'un projet FEDER autour de l'image patrimoniale en lien avec la MSHS. Son activité de recherche se concentre sur la recherche de zones saillantes dans les images et les vidéos et leur prise en compte dans les algorithmes développés pour les applications d'indexation.

\section{CHRISTINE FERNANDEZ-MALOIGNE}

Christine Fernandez-Maloigne est Professeur en Traitement du Signal et des Images depuis 1998 à l'Université de Poitiers, où elle est Vice-Président en charge des relations internationales depuis juin dernier. Elle a créé en 2000 l'équipe ICONES, qui fait aujourd'hui référence, aux niveaux national et international, en imagerie couleur, au sein du Laboratoire XLIM dont elle a été directrice adjointe de janvier 2012 à juin 2016. Elle est également, depuis 2013, directrice de la fédération de recherche CNRS MIRES qui regroupe tous les laboratoires Math-STIC dans l'ex PRES Limousin, Poitou-Charentes. À ce titre, elle est toujours porteuse, pour 2015-2020, d'un programme du CPER de l'ex région Poitou-Charentes, maintenant Nouvelle Aquitaine, portant sur les usages du numérique (et notamment des images numériques) pour le citoyen; ce programme associe 17 laboratoires en Math, STIC, SPI, SHS et droit. Au niveau international, elle est secrétaire de la Division 8, «Image Technology » de la CIE, Commission Internationale de l'Éclairage, organisme de normalisation; et Éditrice en chef associée du journal JOSA A, spécialisée sur la thématique images et couleur numériques. 